\title{
Thermal effects during elastic and plastic deformation of structural steel
}

\author{
by E.S. Lukin, A.M. Ivanov
}

Dept. of Modeling and Fracture, Institute of Physical-Technical Problems of the North SB RAS, Russia, lukin@iptpn.ysn.ru

\begin{abstract}
The results of the thermovision investigation of the elastic and the plastic deformation kinetic of constructional steels are presented. Plain specimens and specimens with central whole stress concentration subjected by one-axis static strain using the thermovision cam TKVr-IFP are investigated. The thermograms of specimens was corresponding a different stages of deformation are presented. Peculiarities of plastic deformation kinetics are determined on the basis of the analysis infrared emission patterns. Investigation of thermal effects during the deformation of structural steel is possessed to determine the limit condition of material.
\end{abstract}

\section{Introduction}

Deformation of structural steels is accompanied by different thermal effects. This is the elastic and plastic thermal effects. The above effects following the elastic and plastic deformation are known beginning the middle of XIX century. J.P.Joule has discovered that at tension a metal bar is cooled slightly, at fast unloading - is heated and at the instantaneously application of the compressive load a short metal bar is slightly heated. Heating and cooling of elastic bodies at loading have been experimentally investigated and described by W. Thomson (Kelvin). A substantial number of papers devoted to the study of the change in temperature, following the processes of deformation of solids, with the use of thermoelectric, calorimetric and thermovision methods are performed in the XX century [1-3].

At the elastic tensile deformation one observes cooling of a metal, while at the elastic compressive - it's heating. Transition to the plastic stage of the deformation process is followed by irreversible plastic deformation, release and dissipation energy in the form of heat.

The existence of modern temperature measuring systems makes it possible to analyze thermal images in a real time regime with the sufficiently high thermal sensitivity and determine changes in the temperature of the sample under study in time. Thermal fields of the objects, locally heated during plastic deformation, are shown in the form of thermograms of thermal maps. Unlike the contact methods of measuring the temperature in a point, for example with the use of thermocouples, non-contacting method records the thermal field of the whole surface under study. The advantages of thermovision method are as follows: substantially high thermal sensitivity, the absence of the contact, temperature measurement in any point and region, recording of thermograms of the object (thermal field all over surface) in real time (in the regime of the thermofilm), construction of temperature distribution along section and saving of the thermograms and thermofilm in the form of files [4].

\section{The materials and instruments tested}

The standard sheet specimens from the steels Fe360 and 09G2S were subjected to static tensile test at rate of deformation $\dot{\varepsilon}=2.4 \times 10^{-3} \mathrm{~s}^{-1}$. The chemical composition of the steel Fe360 was $0.15 w t \%$ C, 0.2 wt \% Si, 0.52 wt \% Mn, $0.17 w t \% \mathrm{Cr}, 0.13 w t \% \mathrm{Ni}$, and $0.25 w t \% \mathrm{Cu}$ and steel 09G2S was $0.12 w t \% \mathrm{C}, 0.8 w t \% \mathrm{Si}, 1.7 w t \% \mathrm{Mn}, 0.3 w t \%$ $\mathrm{Cr}, 0.3 \mathrm{wt} \% \mathrm{Ni}, 0.3 \mathrm{wt} \% \mathrm{Cu}$. Tensile tests were performed on the "UTS-20k" and Instron-1195 testing machines with a constant strain rate. The yield strength and the ultimate strength of steel Fe360 were $330 \mathrm{MPa}$ and $510 \mathrm{MPa}$. And for the steel 09G2S - $330 \mathrm{MPa}$ и $460 \mathrm{MPa}$, respectively.

For the experimental study of deformation and fracture kinetics of smooth samples and samples with stress concentrators we have used the thermocam "TKVr-IFP" produced by the Institute of Physics of Semiconductors SSB RAS. Limiting sensitivity $\pm 0.028 \mathrm{~K}$, frame frequency -20 frames/second.

During the experiments the analysis in the regime of obtaining the static thermograms and in the regime of real time with the representation of thermal maps of sample on monitor of the computer has been performed. Single thermograms and thermofilms are saved in the form of files.

The stage development of deformation is studied according to thermograms and the temperature diagram superposed with the diagram of tension. The scheme of performing the thermovision measurements stipulated the maximum possible space resolution.

\section{Experimental results}


Thermograms of tests under the condition of homogeneous stressed-strained state are presented. The thermograms (infrared radiation patterns) characterizing kinetics of thermal field development alone the sample and corresponding to different stages of deformation. Dynamics of the thermal field development represents the processes of plastic deformation and their localization. The occurrence of the local zone of the infrared glow and distribution of the thermal field front alone the sample correspond to the yield site where the advance of Chernov-Luders bands is observed.

To the end of the yield site the thermal front reaches the opposite end of the sample. Knowing the rate of the distribution of the thermal field front one can judge about the dynamics of the movement of a deformation site in the process of plastic deformation. The occurrence of local zone of the intensive infrared glow corresponds to initiation of "narrowing" in the sample. During rupture of the sample one can observe a bright burst of the infrared radiation.
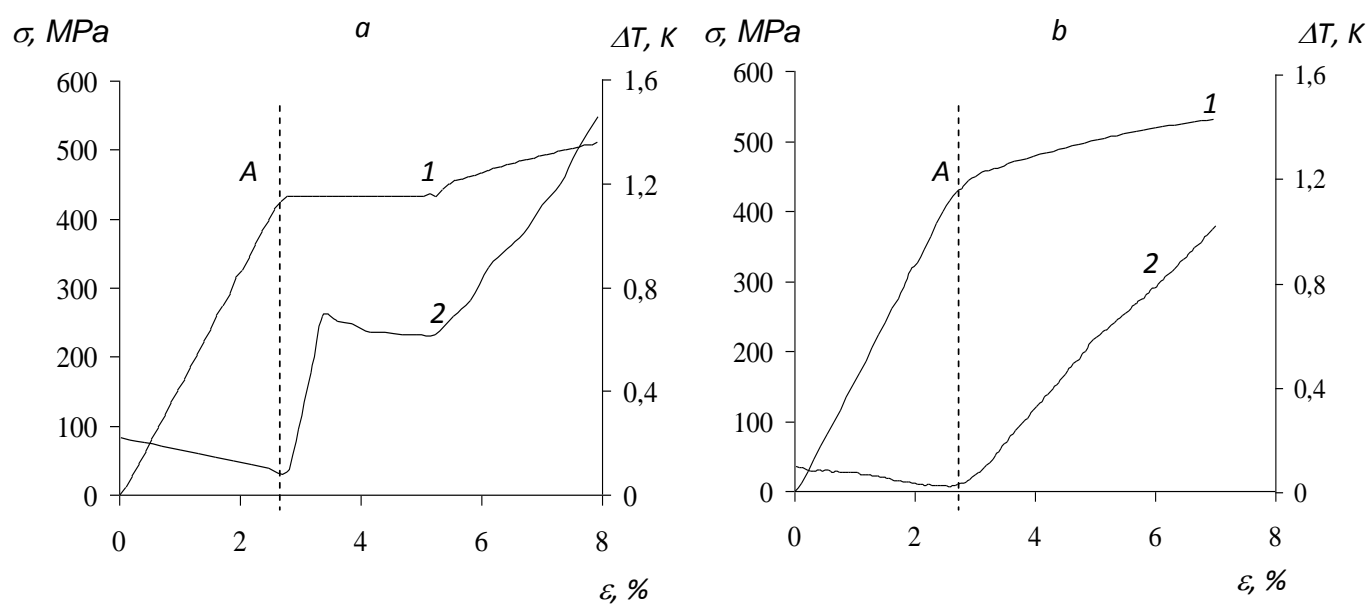

Fig. 1. Diagram of deformation 1, combined with temperature change 2 of steel verses elongation $\varepsilon$, $a$ - with visual yield stress, $\mathrm{b}$ - without visible yield stress

The dependence of the temperature change during deformation of sample is presented on Figure 1. At the elastic stage of deformation the temperature drop is observed (before line $A$ on the curve 2). The yield site is characterized by the rapid temperature risen (corresponding line $A$ on curve 2). Temperature gradient on curve 2 corresponding the moment of the beginning of plastic deformation.

\section{Conclusion}

The thermovision method possesses to investigate kinetics of elastic and plastic deformation of steel. By the thermograms can be determine the beginning of local yielding. Analyze of temperature change during deformation of steel possess to find the yielding moment. The onset of limitation states on the macromechanical level, independently of differences in the mechanisms of deformation and development of damages, one can determine by characteristic changes in the temperature of sample heating.

\section{Acknowledgments}

This work has been supported by the Russian Foundation for Basic Research under Grant RFBR No. 12-01-98509.

\section{REFERENCES}

[1] Thompson (Lord Kelvin). On the Dynamical theory of heat // Transactions of the Royal Society of Edinburgh, Vol. 20, 1853, PP. 261-283.

[2] Taylor G.I., Quinney H. The latent energy remaining in a metal after cold working. //Proc. Roy. Soc., 1934, vol. CXLIII.-A., P. 307-326.

[3] D. Oliver. Analysis of the stress field by using thermal radiation // Cobayashi. Book 2. M.:Mir, 1990. - $552 \mathrm{c}$.

[4] Pieczyska E.A., Gadaj S.P., Nowacki W.K. Thermoelastic and thermoplastic effects during loading and unloading of austenitic steel // Quantitative infrared thermography 4, QIRT'98, Proceedings of Eurotherm Seminar No. 60. - Lodz, Poland, 2000. - PP. 112-116. 